\title{
A multiscale approach for precipitation verification applied to the FORALPS case studies
}

\author{
A. Lanciani ${ }^{1,2}$, S. Mariani ${ }^{2,3}$, M. Casaioli ${ }^{2}$, C. Accadia ${ }^{4}$, and N. Tartaglione ${ }^{5}$ \\ ${ }^{1}$ Inter-Universities National Consortium for Physics of Atmospheres and Hydrospheres (CINFAI), Camerino, Italy \\ ${ }^{2}$ Agency for Environmental Protection and Technical Services (APAT), Rome, Italy \\ ${ }^{3}$ Mathematics Department, University of Ferrara, Ferrara, Italy \\ ${ }^{4}$ EUMETSAT, Darmstadt, Germany \\ ${ }^{5}$ Physics Department, University of Camerino, Camerino, Italy
}

Received: 25 August 2007 - Revised: 14 December 2007 - Accepted: 23 January 2008 - Published: 9 April 2008

\begin{abstract}
Multiscale methods, such as the power spectrum, are suitable diagnostic tools for studying the second order statistics of a gridded field. For instance, in the case of $\mathrm{Nu}-$ merical Weather Prediction models, a drop in the power spectrum for a given scale indicates the inability of the model to reproduce the variance of the phenomenon below the correspondent spatial scale. Hence, these statistics provide an insight into the real resolution of a gridded field and must be accurately known for interpolation and downscaling purposes. In this work, belonging to the EU INTERREG IIIB Alpine Space FORALPS project, the power spectra of the precipitation fields for two intense rain events, which occurred over the north-eastern alpine region, have been studied in detail. A drop in the power spectrum at the shortest scales (about $30 \mathrm{~km}$ ) has been found, as well as a strong matching between the precipitation spectrum and the spectrum of the orography. Furthermore, it has also been shown how the spectra help understand the behavior of the skill scores traditionally used in Quantitative Precipitation Forecast verification, as these are sensitive to the amount of small scale detail present in the fields.
\end{abstract}

\section{Introduction}

Mesoscale meteorology, dealing with phenomena ranging from rain bands to thunderstorms, is deeply concerned with small scale detail. Nowadays Numerical Weather Prediction (NWP) models are capable of producing such detail. However, their verification is nontrivial. For instance, traditional skill scores (which measure point-to-point matching) are sensitive to small displacement errors, resulting in a double penalty effect, so that increasing forecast detail

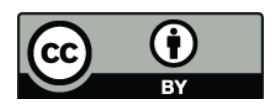

Correspondence to: A. Lanciani

(alexandre.lanciani@apat.it) may decrease the score. This kind of first order verification can be deceptive. Higher order moments must be studied to make sure that the fields being compared are defined on grids with the same real resolution, and even if they are, that they have the same amount of small scale detail (see, e.g., Beck and Ahrens, 2004; Chèruy et al., 2004; Grasso, 2000; Harris et al., 2001; Tartaglione et al., 2002).

In this work, performed within the EU INTERREG IIIB Alpine Space FORALPS project (http://www.foralps.net), we have studied in detail the power spectra of the precipitation fields for two intense rain events which occurred over the north-eastern alpine arc. The first event took place on 16-20 November 2001, whilst the second one occurred on 8-10 September 2005. Namely, we have studied the spectra of three operational Limited Area Models' (LAMs) forecasts, and attempted to compare these results with those from a Quantitative Precipitation Forecast (QPF) intercomparison study by means of traditional skill scores (Mariani and Casaioli, 2008). We have also investigated how these spectra are affected by different interpolation techniques that are usually employed in QPF verification.

The rest of the paper is arranged as follows: in the next section we will define the methodology, then we will describe the observations and models data set. Afterward we will discuss some results we have obtained, and finally we will draw some conclusions.

\section{Methodology}

\subsection{Power Spectrum}

The power spectrum (Wilks, 1995) can be an effective diagnostic tool to study the higher order moments of a gridded field and its scale dependency (Goody et al., 1998). The 2-D spectrum $E\left(k_{x}, k_{y}\right)$ of a real field $\phi(x, y)$, where $k_{x}$ and $k_{y}$

Published by Copernicus Publications on behalf of the European Geosciences Union. 


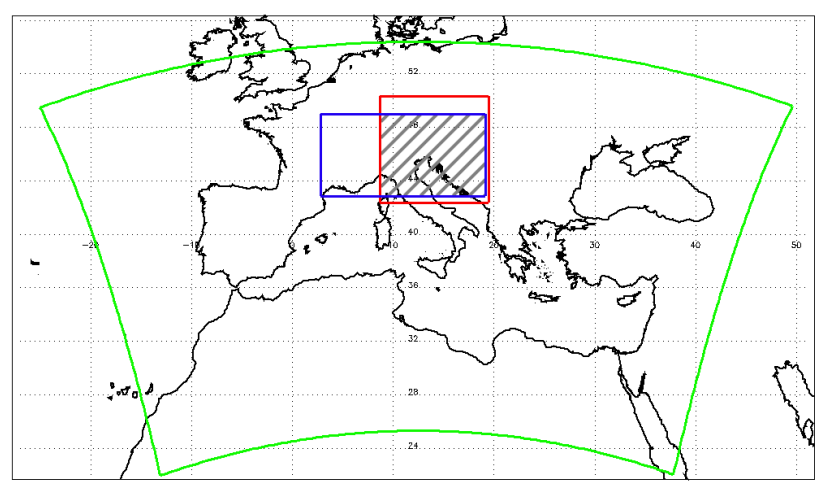

Fig. 1. LAMs' domains: ALADIN (red), QBOLAM (green), and WRF (blue). The gray shaded area is the verification area.

are the wavenumber components, is formally defined as the Fourier transform of the autocorrelation function:

$E\left(k_{x}, k_{y}\right)=\frac{1}{\sqrt{2 \pi}} \int d l_{x} d l_{y} e^{-i\left(k_{x} l_{x}+k_{y} l_{y}\right)} K\left(l_{x}, l_{y}\right)$,

where

$K\left(l_{x}, l_{y}\right)=\int d x d y \phi\left(x+l_{x}, y+l_{y}\right) \phi(x, y)$,

is the autocorrelation function and $l_{x}$ and $l_{y}$ are respectively the lag in the $x$ direction and in the $y$ direction.

However, it can also be computed according to the WienerKhinchin theorem by multiplying the 2-D Fourier transform by its complex conjugate:

$E\left(k_{x}, k_{y}\right)=\frac{1}{2 \pi}\left|\int d x d y e^{-i\left(x k_{x}+y k_{y}\right)} \phi(x, y)\right|^{2}$.

We have chosen the latter method, as it suppresses some computational noise. Moreover, a Hanning window was previously used to filter the data and to reduce aliasing (Press et al., 1992).

The relationship between the model domain grid size $\Delta$ and the wavenumber grid size $\tilde{\Delta}$ is given by $\tilde{\Delta}=(N \Delta)^{-1}$, where $N$ is the number of the model grid points. The largest wavenumber from which we can extract meaningful information is given by the Nyquist frequency $(2 \Delta)^{-1}$. Since the models are defined on different grids with different grid steps, the wavenumber ranges will be different, too. The 2-D spectrum can be presented as an isotropic power spectrum $E(k)$, if it is averaged angularly and $k$ is defined as $k=\sqrt{k_{x}^{2}+k_{y}^{2}}$. The width of the bands where the average is made is chosen in order to smooth the isotropic spectrum without losing any significant information.

Scaling of the power spectrum occurs when it can be written as $E(k) \sim k^{-\beta}$. In other words, the spectrum shows scale invariance if it is linear in $k$ on a log-log plot. The absolute value of the spectral slope, that is, $\beta$, is an indicator of the fields smoothness. The higher $\beta$, the smoother, more organized, is the structure.

\subsection{QPF Verification}

In this study, for QPF verification, we have used the equitable threat score (ETS; Schaefer, 1990). This score is tallied up on $2 \times 2$ contingency tables (Wilks, 1995), which summarize in a categorical way possible combinations of forecast and observed events above or below a given precipitation threshold. For each selected threshold, four categories are then defined: hits; false alarms; misses and correct non-rain forecasts ( $a, b, c$ and $d$ respectively). To reduce the sensitivity of skill scores to small changes into the population of the contingency table elements, they have been calculated on a sum of daily contingency tables depending on the time period considered. Thus, for the November 2002 event scores have been calculated on five contingency tables, whereas for the September 2005 event they have been calculated over three tables.

The ETS is an accuracy measure for events, that is, it measures how well the forecast "yes" events correspond to the observed "yes" events. ETS allows also for the number of hits that would be obtained purely by chance (random forecast). It is defined by:

$\mathrm{ETS}=\frac{a-a_{r}}{a+b+c-a_{r}}$,

where $a_{r}=(a+b)(a+c) /(a+b+c+d)$ is the number of model hits expected from a random forecast.

A score equal to one represents a perfect score; whilst a value close to zero or negative means that the model has a questionable forecasting ability.

\section{Models data set and observations}

\subsection{Models}

Forecasts were modelled by three LAMs (whose domains are shown in Fig. 1) the 11-km Aire Limitée Adaptation dynamique Développement InterNational (ALADIN; http: //www.cnrm.meteo.fr/aladin/), operational at the Environmental Agency of the Republic of Slovenia (EARS); the $0.1^{\circ}$ QUADRICS BOlogna LAM (QBOLAM) operational at the Agency for Environmental Protection and Technical Services (APAT; Speranza et al., 2007); the hydrostatic version of the 10-km Weather Research and Forecasting (WRF; http://wrf-model.org/) model running in a research configuration at the Regional Meteorological Observatory of FriuliVenezia Giulia (OSMER). Moreover, the $0.5^{\circ}$ T511 L60 ECMWF global model (http://www.ecmwf.int/) is also used in the comparison.

The models differ in parameterization and discretization schemes (ALADIN and ECMWF are spectral models, while QBOLAM and WRF are finite difference models) and in both initial and boundary conditions. Global analyses and forecasts from ECMWF are employed as initial and boundary conditions, respectively, by QBOLAM and WRF, whereas 


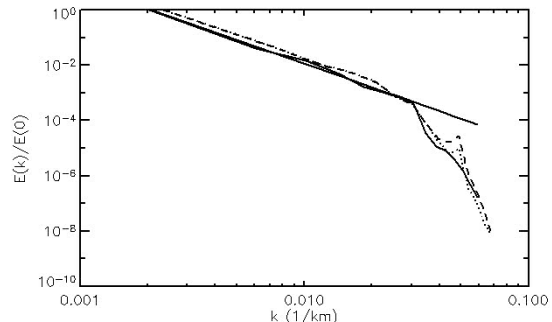

(a) ALADIN

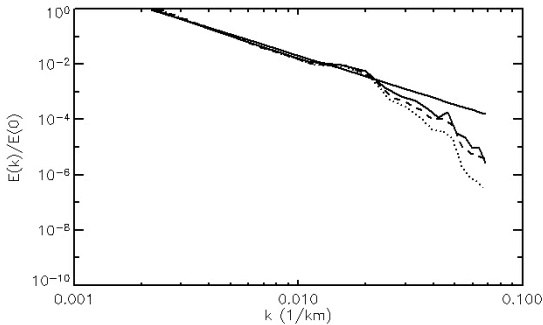

(b) QBOLAM

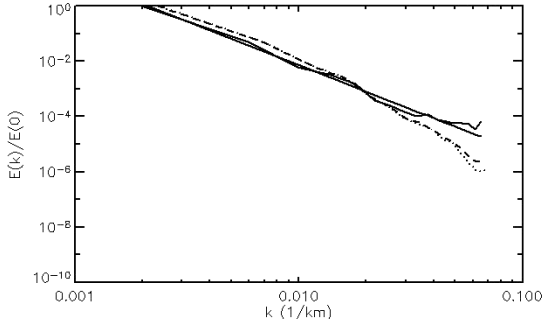

(c) WRF

Fig. 2. Power spectrum of the 16 November $200224 \mathrm{~h}$ accumulated precipitation forecast: original grid (solid line), bilinear interpolation (dotted line) and remapping (dashed line). A linear fit on the first part of the original spectrum is also shown.

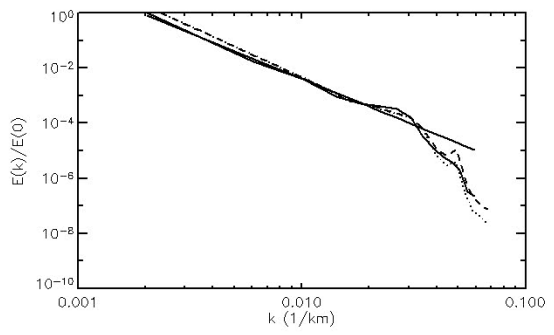

(a) ALADIN

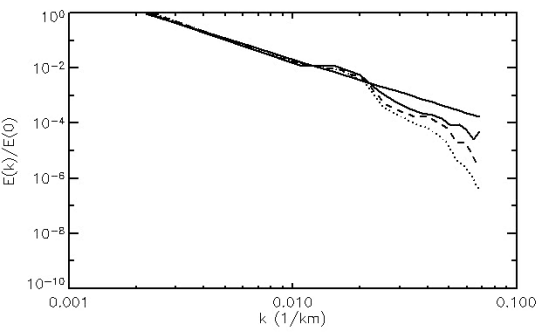

(b) QBOLAM

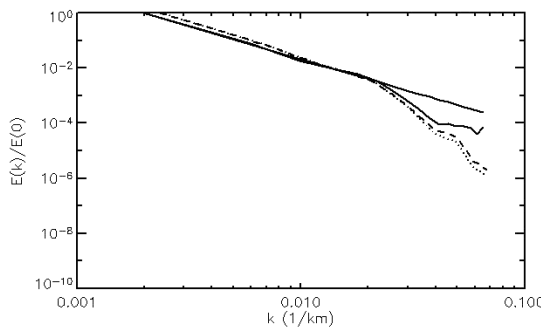

(c) WRF

Fig. 3. Same as Fig. 2, but for the 9 September 2005 forecast.

ALADIN employs ARPEGE (Action de Recherche Petite Echelle Grande Echelle; http://www-pcmdi.llnl.gov/) analyses and forecasts. Moreover, $24 \mathrm{~h}$ ALADIN runs, starting at 00:00 UTC, are used, while the other two LAMs are initialized at 12:00 UTC of the previous day for a $36 \mathrm{~h}$ run, and the first $12 \mathrm{~h}$ of each run are discarded as a spin-up.

The models considered differ also in horizontal grid size. Since the intercomparison results may be sensitive to such difference, precipitation forecasts have been also postprocessed on two common verification grids (with grid size of $0.1^{\circ}$ and $0.5^{\circ}$ respectively) by means of bilinear interpolation and also remapping ${ }^{1}$ (e.g., see Accadia et al., 2003), and accumulated at $24 \mathrm{~h}$, starting from 00:00 UTC.

All these differences notwithstanding, all the models (including the hydrostatic WRF) consider precipitation a diagnostic variable. As such, it is calculated after all the prognostic variables have been advected. This is particularly important for ALADIN, because as a spectral model its dynamics take place in spectral space, whereas the microphysics take place in physical space.

\footnotetext{
${ }^{1}$ Remapping is performed by subdividing each verification grid box into $n \times n$ sub-boxes (in this case, $n=13$ ). Then the value of the nearest native grid point is assigned to each sub-grid point. The average of these sub-grid point values produces the remapped value of the verification grid point.
}

\subsection{Observations}

For the November 2002 event, precipitation data have been obtained from 766 working rain gauges belonging to the networks of APAT [former Italian National Hydrographic and Marigraphic Service (SIMN) network], Agenzia Regionale per la Protezione dell'Ambiente (ARPA) of EmiliaRomagna, ARPA of Liguria, OSMER, EARS and ZentralAnstalt für Meteorologie und Geodynamik (ZAMG).

For the September 2005 event, precipitations have been collected from 781 working rain gauges of APAT, ARPA of Emilia-Romagna, ARPA of Liguria, ARPA of Lombardia, OSMER, EARS, and ZAMG.

In order to produce an adequate 24-h observed rainfall gridded analysis over the two common verification grids, a two-pass Barnes objective analysis scheme has been used (Barnes, 1964, 1973). This is a Gaussian weighted-averaging technique that assigns a weight to each rain gauge observation as a function of the distance between the gauge and the grid box center.

More precisely, call $x$ the position of the analysis point and $x_{k}, k=1, \ldots, K$, the positions of the gauges within its region of influence. A first pass is performed to produce a 


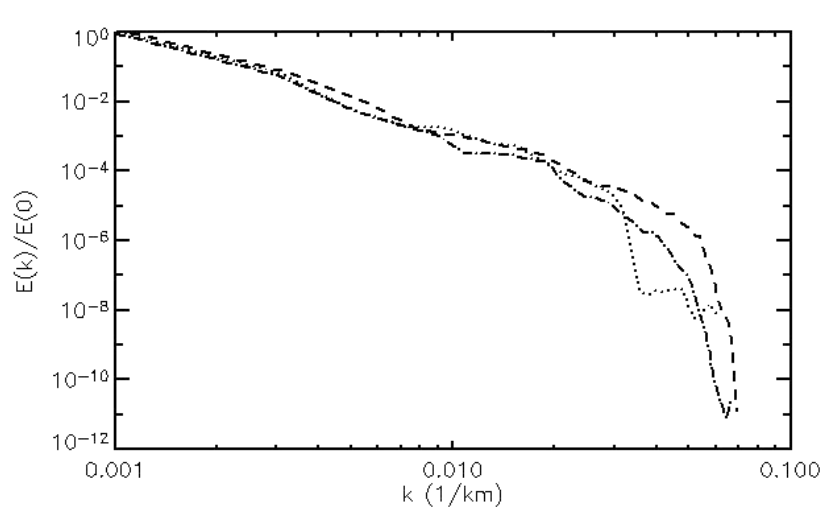

Fig. 4. Spectra of the models' orography: ALADIN (dotted line), QBOLAM (dashed line) and WRF (dot-dashed line).

first-guess precipitation analysis (Daley, 1991):

$f_{A}^{(0)}(x)=\sum_{k=1}^{K} w_{k} f_{O}\left(x_{k}\right)$

where $f_{O}\left(x_{k}\right)$ is the precipitation measured at the $k$-th gauge and the weights are defined as

$w_{k}=\exp \left(-\frac{\left|x_{k}-x\right|^{2}}{4 a R^{2}}\right)$.

In the previous equation $\left|x_{k}-x\right|$ is the distance between the analysis point and the position of the gauge, $R$ is the average data spacing and $a$ is a proportionality coefficient that results in the optimal response function.

This is followed by a second pass that is needed to increase the amount of detail in the first guess:

$f_{A}^{(1)}(x)=f_{A}^{(0)}(x)+\sum_{k=1}^{K} w_{k}\left[f_{O}\left(x_{k}\right)-f_{A}^{(0)}\left(x_{k}\right)\right]$.

The algorithm's convergence is very fast: Koch et al. (1983) have shown that only these two passes through the data are needed to achieve the convergence of the analysis to the observations, provided that a numerical convergence parameter $\gamma \in(0,1)$ is used to redefine $R$ in Eq. (6) as $R^{\prime}=\gamma R$ in the second pass. We have used $\gamma=0.3$ and $R=0.15^{\circ}$.

\section{Results}

The spectra were calculated, using the IDL ${ }^{2}$ software and its Fast Fourier Transform (FFT) and Hanning algorithms, over the intersection of all the models' domains, i.e.: between $8.7^{\circ} \mathrm{W}$ and $18.4^{\circ} \mathrm{W}$ in longitude and $42.9^{\circ} \mathrm{N}$ and $48.9^{\circ} \mathrm{N}$ in latitude (see the shaded area in Fig. 1).

\footnotetext{
${ }^{2}$ Interactive Data Language, version 6.4, (C) ITT Visual Information Solutions, Boulder, CO, http://www.ittvis.com/.
}

The spectrum of the fields on their native grids displays scale invariance down to about $30 \mathrm{~km}$, after which there is a fall off (we show two examples in Figs. 2 and 3). Hence, $30 \mathrm{~km}$ can be taken as the minimal resolution of the grids insofar as the precipitation is concerned. Actually, taking into account numerical implementation issues, such as superdiffusion operators that ensure computational stability, it is probably even lower (for a more throughout discussion on effective model resolution, see Frehlich and Sharman, 2007, and the references therein).

Both interpolation methods result in smoother fields, although bilinear interpolation slightly more so. This is more striking in the case of QBOLAM (see Figs. 2b and 3b).

The correlation between the precipitation spectra on the models' native grid and the spectra of the models' orography is very high, always higher than 0.98 . But it is perhaps more interesting to note that the spectrum of ALADIN's orography drops sharply for scales smaller than 3 grid steps (Fig. 4). Orography is spectrally represented in ALADIN, which is why its power spectrum should be equal to zero below about $30 \mathrm{~km}$. The residual power arises from back transformation and interpolation to the physical grid.

It is interesting at this point to consider also the ETS score (Fig. 5). Here we will only discuss the 2002 case. The greatest difference is on 18 November 2002 (see also Fig. 6 for an eyeball comparison between the forecast fields and the observation field). As can be seen from Fig. 5a, ALADIN appears to have a better ETS than the other two models, at least up to

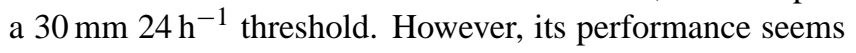
to worsen if we look at the $0.1^{\circ}$ grid (Fig. 5b).

As we anticipated in the introduction, this can be partly explained by the different spectral behavior of the models. Indeed, the values of $\beta$ for both events can be obtained from a linear fit on the first (scaling) part of the spectra. At least for the 2002 event, ALADIN's forecast on the original domain always has more structure than WRF's, which in turn has more structure than QBOLAM's. This would seem to be in contradiction with our earlier discussion on the orography spectrum. The contradiction could be explained by several reasons, both physical (absence of prognostic microphysical fields implies that none of the orographic rain lands in the lee of the mountains, amplifying small scale features; Dr. Mark Žagar, 2007, personal comunication) and numerical (spectral truncation entails a Gibbs effect, see Lindberg and Broccoli, 1996). However, this aspect needs further study to be fully understood.

Moreover, if we look at the spectra of the fields remapped on the same $0.1^{\circ}$ and $0.5^{\circ}$ common grids, where the skills scores were calculated (see Figs. 7 and 8), we find that the differences among the models' spectra are greater on the former grid than on the latter.

Accordingly, ALADIN's ETS is more penalized on the $0.1^{\circ}$ grid and the intercomparison should not be performed there but rather on the $0.5^{\circ}$ grid. On the other hand, from Fig. 5a we see that the models' scores are much more 


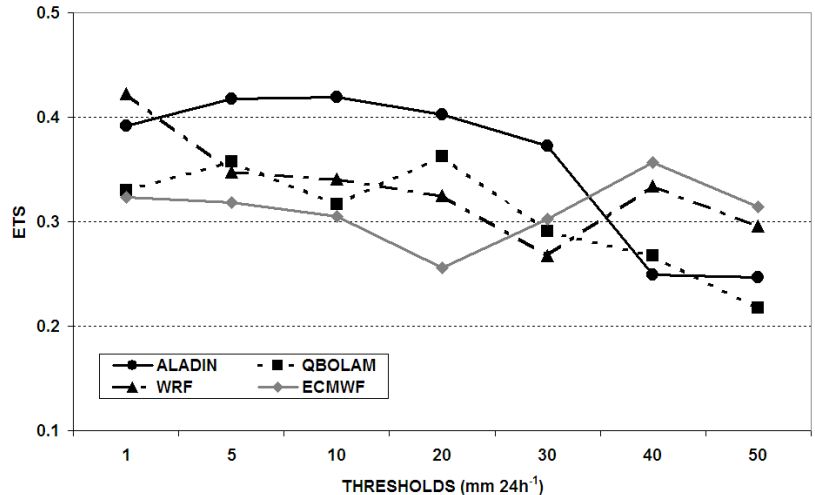

(a) $0.5^{\circ}$ verification grid

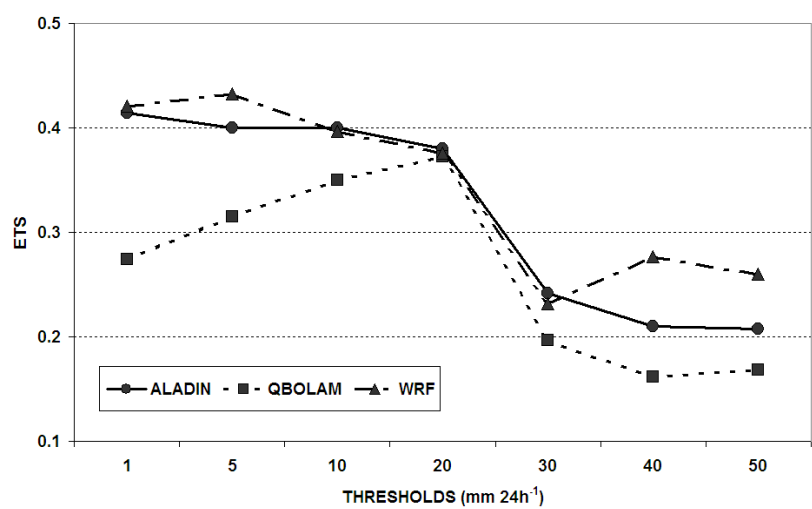

(b) $0.1^{\circ}$ verification grid

Fig. 5. The ETS score for the 2002 event reported as a function of the selected thresholds.
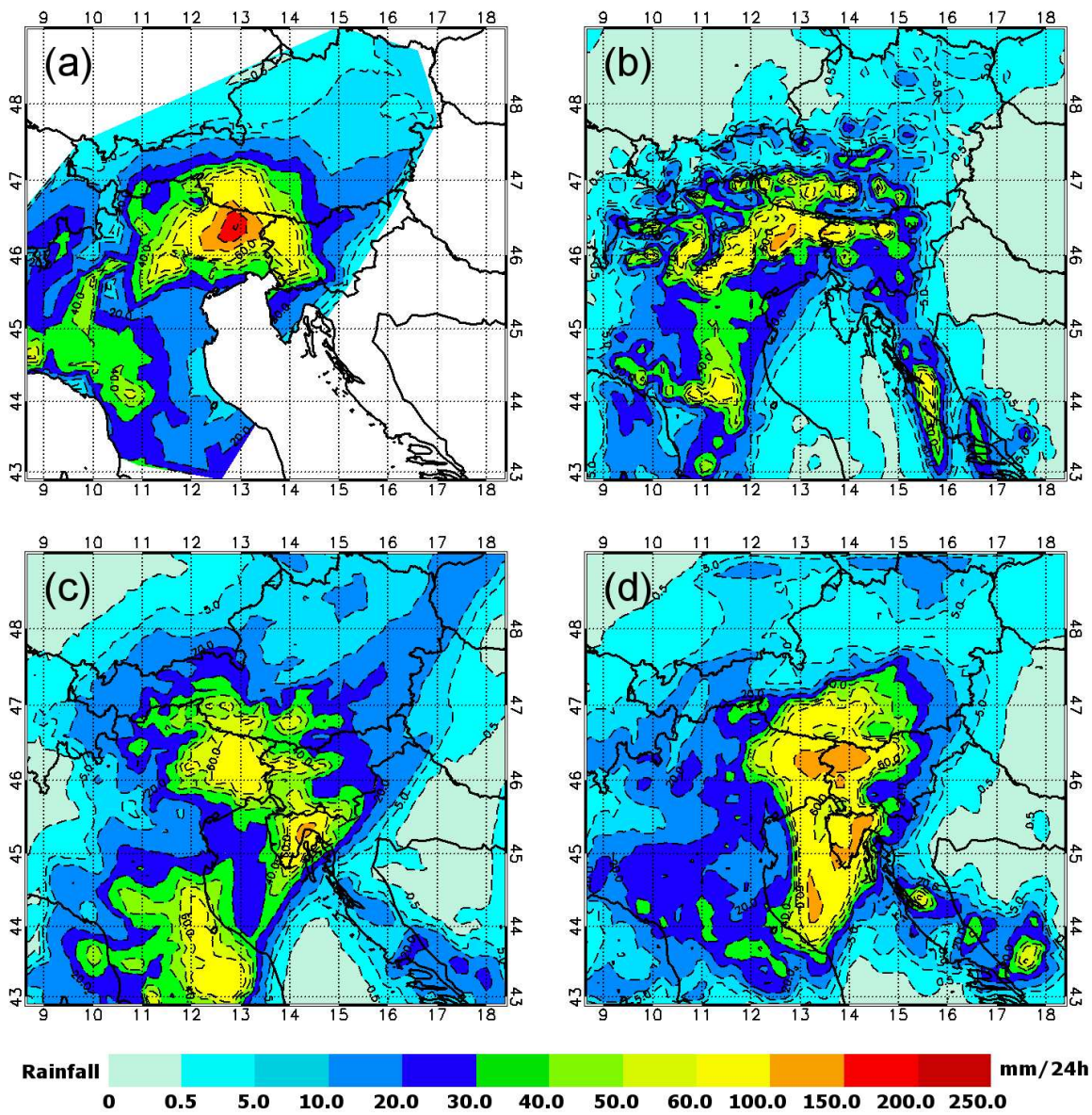

Fig. 6. Contours (in mm $24 \mathrm{~h}^{-1}$ ) of precipitation observed (a) and forecast by ALADIN (b), QBOLAM (c) and WRF (d) on 18 November 2002. Forecasts are remapped on the $0.1^{\circ}$ common verification grid. For the observations, the contours of the $0.1^{\circ}$ Barnes analysis is masked over the sea. 


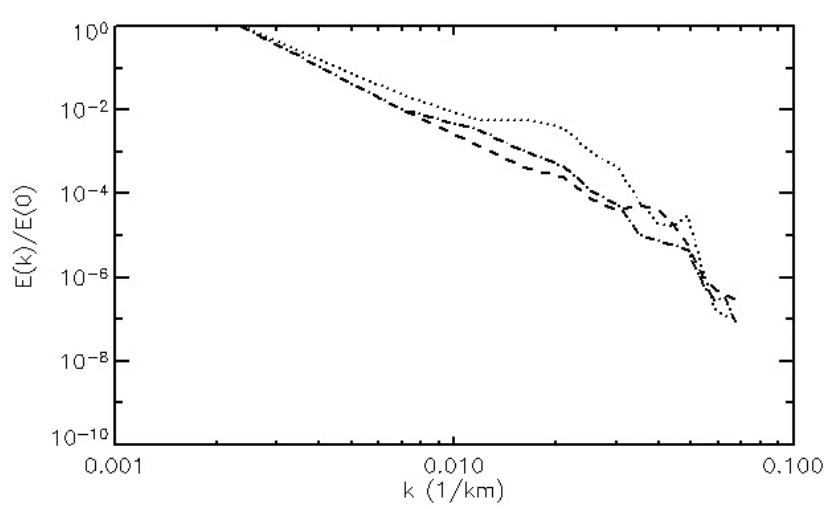

Fig. 7. Spectra of the 18 November $200224 \mathrm{~h}$ accumulated precipitation interpolated on a $0.1^{\circ}$ common grid: ALADIN (dotted line), QBOLAM (dashed line) and WRF (dot-dashed line).

homogeneous, although ALADIN seems to behave slightly better. In a similar way, the coarser field is more penalized as the threshold increases, and this is again reflected in the ETS behavior - and, one more time, the difference is more macroscopic on the $0.1^{\circ}$ grid than on the $0.5^{\circ}$ grid, which is again coherent with our results on the spectra.

That increasing the threshold entails a reduction in the ETS of the more detailed field can be understood in the following way: If the field is not smooth, two neighboring points can have very different values. In particular, the overlap between two slightly shifted peaks, one belonging to the forecast field and the other to the observed field, will be lesser than for a smoother forecast field. Since whatever is below the threshold gets cut, for a sufficiently high threshold what remains in the former case are two isolated peaks, entailing a double penalty error to the ETS. On the contrary, if the forecast is smooth there will still be an overlap resulting in both a false alarm (the forecast peak) but also a hit (where the two fields overlap).

\section{Conclusions}

In this work, we have studied the power spectra of the precipitation fields forecast by three NWP LAMs, namely: ALADIN operational at EARS, QBOLAM operational at APAT and WRF operational at OSMER. For comparison and comprehensiveness we have also considered the ECMWF forecasts and the observations available for the two events: 1620 November 2001, and 8-10 September 2005. We focused on the regions of the Alpine Space as they are the subject of the FORALPS project within which this study was performed. Our results were threefold.

First, we found that there is a drop of the power spectrum at the shortest scales that can be theoretically resolved by these models, confirming that their real resolution is actu-

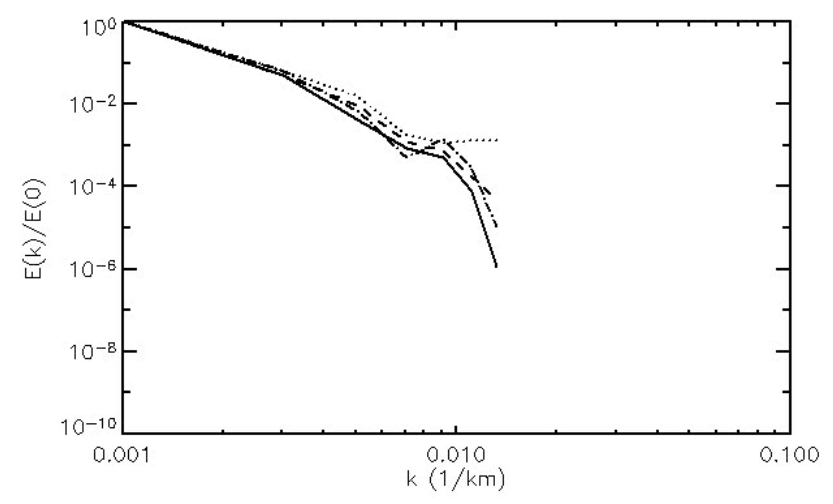

Fig. 8. Spectra of the 18 November $200224 \mathrm{~h}$ accumulated precipitation interpolated on a $0.5^{\circ}$ common grid: ALADIN (dotted line), QBOLAM (dashed line), WRF (dot-dash line) and ECMWF (solid line).

ally coarser than the grid mesh-size. Moreover, interpolation techniques further worsen the resolution by oversmoothing the precipitation fields. Between the two techniques we considered, that is, remapping and bilinear interpolation, the latter fares the worst. This first result is consistent with previous studies on the subject.

Second, we showed that there is a strong matching between the precipitation spectra and the spectra of the orography used in the models. Although this should not come as a surprise, as the two events we studied have a clear orographic component, it is important to recognize this aspect explicitly. Indeed, in this study precipitation is a diagnostic variable, deriving from complex processes in NWP models which involve specific humidity and temperature. The two latter variables are prognostic variables and as such their equations include, for reasons owning to numerical stability, diffusion operators that cause a spectral drop. Instead, a clear and direct action of diffusion operators on precipitation has still to be fully understood.

Finally, we have related the spectra of the fields with the ETS, one of the traditional QPF verification skill scores that are often used in model intercomparison. As we expected, the lesser smoothness of ALADIN's field was penalized by the ETS on the higher resolution (in fact, unrealistically high resolution - see above) grid. This suggests that for QPF verification purposes the comparison should be performed on the coarser grid only. Otherwise particular attention must be paid in the discussion of the results, namely with regard to which model performs better. Even so, a comparison at a finer resolution can provide physical insight into the model behavior, such as ALADIN's tendency to produce small scale features. ALADIN's ETS was further penalized when the thresholds in the contingency table calculation became very high.

It should be noted that, as in any other work restricted to case studies, what we have analyzed here are merely 
snapshots. The subject of precipitation second order statistics deserves further study. For example, other multiscale methods (e.g., wavelet decomposition) could be used. Perhaps more importantly one could try to understand the physical and mathematical reasons behind our results. Further studies along this direction, that include the use of the observations' spectrum, are on the way.

Acknowledgements. This work was developed and funded under the framework of the EU INTERREG IIIB Alpine Space FORALPS project (contract n. I/III/3.1/21). We thank the FORALPS partners ARPA of Lombardia, EARS, OSMER and ZAMG for providing us the observed and forecast data used in this study. Our thanks also go to the ARPA of Emilia-Romagna and of Liguria for precipitation data over their territory. Finally, we wish to thank B. Lastoria (APAT) for the help on data processing, the two anonymous reviewers for their suggestions and, last but not least, A. Speranza (University of Camerino) and G. Monacelli (APAT) for the support.

Edited by: S. C. Michaelides

Reviewed by: two anonymous referees

\section{References}

Accadia, C., Mariani, S., Casaioli, M., Lavagnini, A., and Speranza, A.: Sensitivity of Precipitation Forecast Skill Scores to Bilinear Interpolation and a Simple Nearest-Neighbor Average Method on High-Resolution Verification Grids, Weather Forecast., 18, 918-932, 2003.

Barnes, S. L.: A technique for maximizing details in numerical weather map analysis, J. Appl. Meteorol., 3, 396-409, 1964.

Barnes, S. L.: Mesoscale objective analysis using weighted timeseries observations, p. 60, tech. memo. ERL NSSL-62, 1973.

Beck, A. and Ahrens, B.: Multiresolution evaluation of precipitation forecasts over the European Alps, Meteorologische Zeitschrift, 13, 55-62, 2004.

Chèruy, F., Speranza, A., Sutera, A., and Tartaglione, N.: Surface winds in the Euro-Mediterranean area: the real resolution of numerical grids, Ann. Geophys., 22, 4043-4048, 2004,

http://www.ann-geophys.net/22/4043/2004/.
Daley, R.: Atmospheric data analysis, Cambridge University Press, 1991.

Frehlich, R. and Sharman, R.: The use of structure functions and spectra from numerical model output to determine effective model resolution, Mon. Wea. Rev., in press, 2007.

Goody, R., Anderson, J., and North, G.: Testing Climate Models: An Approach, Bull. Am. Meteor. Soc., 9, 2541-2549, 1998.

Grasso, L. D.: The differentiation between grid spacing and resolution and their application to numerical modeling, Bull. Am. Meteorol. Soc., 81, 579-580, 2000.

Harris, D., Foufoula-Georgiou, E., Droegemeier, K. K., and Levit, J. J.: Multiscale statistical properties of a high-resolution precipitation forecast, J. Hydrometeor., 2, 406-418, 2001.

Koch, S. E., desJardins, M., and Kocin, P. J.: An interactive Barnes objective map analysis scheme for use with satellite and conventional data, J. Climate Appl. Meteor., 22, 1487-1503, 1983.

Lindberg, C. and Broccoli, A. J.: Representation of topography in spectral climate models and its effect on simulated precipitation, J. Clim., 9, 2641-2659, 1996.

Mariani, S. and Casaioli, M.: Forecast verifivication: A summary of common approaches and examples of application, FORALPS Technical Report 5, Univerità degli Studi di Trento, Dipartimento di Ingegneria Civile e Ambientale, Trento, Italy, 60 pp., 2008.

Press, W. H., Flannery, B. P., Teukolsky, S. A., and Vetterling, W. T.: Numerical Recipes in FORTRAN: The Art of Scientific Computing, Cambridge University Press, 1992.

Schaefer, J. T.: The critical success index as an indicator of warning skill, Weather Forecast., 5, 570-575, 1990.

Speranza, A., Accadia, C., Mariani, S., Casaioli, M., Tartaglione, N., Monacelli, G., Ruti, P. M., and Lavagnini, A.: SIMM: An integrated forecasting system for the Mediterranean Area, Meteorol. Appl., 14, 337-350, 2007.

Tartaglione, N., Lanciani, A., and Speranza, A.: Analysis of the effects of numerical diffusion on some meteorological fields forecasted by NWP models in the Mediterranean area, in: Proceedings of the 4th European Geophysical Soc. Plinius Conference on "Mediterranean Storm", Mallorca, Spain, 3-5 October 2002, 4 pp., 2002.

Wilks, D. S.: Statistical methods in the atmospheric sciences: an introduction, Academic Press, San Diego (USA), 1995. 